\section{Buchrezension zu: Mutschler Arzneimittelwirkungen}

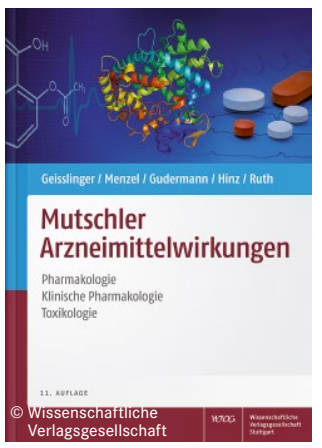

Mutschler Arzneimittelwirkungen

Pharmakologie - Klinische

Pharmakologie - Toxikologie

Gerd Geisslinger, Thomas

Gudermann, Burkhard Hinz, Sabine Menzel und Peter Ruth

1296 S., 654 Abbildungen, 316 Tabellen, ca. 1.400 Strukturformeln. Wissenschaftliche Verlagsgesellschaft Stuttgart, 2020. HC, 78,00€

ISBN: 9783804736634

DOI: $10.1007 / \mathrm{s} 12268-020-1327-0$

(C) Der Autor 2020

„Der Mutschler“ ist ein Klassiker unter den PharmakologieLehrbüchern, der jetzt in seiner 11. Auflage erschienen ist. Das Buch wurde vor mehr als 40 Jahren von Herrn Prof. Dr. Dr. h. c. mult. Ernst Mutschler begründet und mit der 11. Auflage in die Hände eines hochkompetenten Teams aus vier international anerkannten Pharmakologen und einer Apothekerin übertragen. Diese Tatsache zeigt, dass es heutzutage nicht mehr möglich ist, das gesamte Fach in der in dem Buch dargebotenen Tiefe als Alleinautor zu präsentieren. Die Aufgabenteilung ist sehr gut gelungen und dafür gratuliere ich den Autoren.

Das Buch hat eine sehr ansprechende und professionelle Aufmachung mit einer robusten Bindung und einem sehr attraktiven Preis. Es ist definitiv ein „Musthave" für jeden Pharmakologen und Apotheker. Die Autoren präsentieren das gesamte Gebiet der Pharmakologie äußerst aktuell, detailreich, kritisch und sehr schön illustriert. Sehr gut ist das aus den Vorauflagen übernommene Konzept, jede Arzneistoffgruppe in einen breiten (patho)physiologischen Zusammenhang zu stellen. Die Strukturformeln vieler Arzneistoffe sind dargestellt. Ebenso werden beispielhafte Handelspräparate genannt und alle relevanten AWMF-Leitlinien berücksichtigt. Das Buch wird durch ein sehr gutes Verzeichnis weiterführender Literatur und ein umfängliches Sachverzeichnis ergänzt. All dies erhöht seinen Wert.

Das Buch eignet sich besonders gut für Dozenten, die für Mediziner, Pharmazeuten und Naturwissenschaftler ein bestimmtes Thema für den Pharmakologieunterricht vorbereiten möchten. Auch Studenten kann es zum profunden Erarbeiten von spezifischen Themen sehr empfohlen werden. Trotz des konsequenten Weglassens obsoleter Inhalte ist "der Mutschler" wegen des groBen Umfangs zur unmittelbaren Examensvorbereitung im Sinne eines Crashkurses nicht gut geeignet. Diese Lücke füllen andere komplementäre Bücher. Das Buch ist im Dezember 2019 erschienen, also ganz kurz nach der Veröffentlichung der neuen Arzneistoffliste des IMPP. Daher konnten diese letzten Neuentwicklungen naturgemäß in der 11. Auflage noch nicht berücksichtigt werden. Ich bin mir aber sicher, dass die Autoren diese Aktualisierung bei der 12. Auflage machen werden.

Roland Seifert,

Medizinische Hochschule Hannover (MHH),

seifert.roland@mh-hannover.de

Funding Open Access funding provided by Projekt DEAL.
Open Access Dieser Artikel wird unter der Creative Commons Namensnennung 4.0 International Lizenz veröffentlicht, welche die Nutzung, Vervielfältigung, Bearbeitung, Verbreitung und Wiedergabe in jeglichem Medium und Format erlaubt, sofern Sie den/die ursprünglichen Autor(en) und die Quelle ordnungsgemäß nennen, einen Link zur Creative Commons Lizenz beifügen und angeben, ob Änderungen vorgenommen wurden. Die in diesem Artikel enthaltenen Bilder und sonstiges Drittmaterial unterliegen ebenfalls der genannten Creative Commons Lizenz, sofern sich aus der Abbildungslegende nichts anderes ergibt. Sofern das betreffende Material nicht unter der genannten Creative Commons Lizenz steht und die betreffende Handlung nicht nach gesetzlichen Vorschriften erlaubt ist, ist für die oben aufgeführten Weiterverwendungen des Materials die Einwilligung des jeweiligen Rechteinhabers einzuholen. Weitere Details zur Lizenz entnehmen Sie bitte der Lizenzinformation auf http://creativecommons.org/licenses / by/4.0/deed.de. 